\title{
Mechatronic Design of a Robot for Upper Limb Rehabilitation at Home
}

\author{
Elio Matteo Curcio, Giuseppe Carbone \\ Department of Mechanical, Energy, and Management Engineering, University of Calabria Via Bucci, Cubo 46C, 87036 Rende, Italy
}

\begin{abstract}
This paper addresses the design of a novel bionic robotic device for upper limb rehabilitation tasks at home. The main goal of the design process has been to obtain a rehabilitation device, which can be easily portable and can be managed remotely by a professional therapist. This allows to treat people also in regions that are not easily reachable with a significant cost reduction. Other potential benefits can be envisaged, for instance, in the possibility to keep social distancing while allowing rehabilitation treatments even during a pandemic spread. Specific attention has been devoted to design the main mechatronic components by developing specific kinematics and dynamics models. The design process includes the implementation of a specific control hardware and software. Preliminary experimental tests are reported to show the effectiveness and feasibility of the proposed design solution.
\end{abstract}

Keywords: bionic robot, design, simulations, parallel robots, upper limb rehabilitation

Copyright $(\odot)$ The author(s) 2021.

\section{Introduction}

Robotic assisted rehabilitation is a rapidly growing field with increasing applications in clinical care, as reported for example in Refs. [1-10]. The development of robots for the rehabilitation of the upper limbs are characterized by a complexity that is increasing with the movement to be performed ${ }^{[1]}$. Most well-known commercial upper limb rehabilitation robots are the MIT-MANUS ${ }^{[2]}$, sold as InMotion ARM (Interactive Motion Technologies, USA) and ARMin (sold as ArmeoPower by Hocoma AG, Switzerland) ${ }^{[3]}$. There are also two other models used in clinics: ArmeoSpring ${ }^{[4]}$ and ReoGo ${ }^{[5]}$. The MANUS is a five-bar SCARA robot with only two translational Degrees of Freedom (DOFs) for the movement of the elbow and forearm. Motorska's ReoGo is a robotic system for upper limb therapy with 3 DOFs. ArmeoSpring is a passive orthosis for upper limb. It offers a large 3D workspace, where one can detect the 3D position and gripping force of the arm. ArmeoPower is a motorized version of ArmeoSpring, with 6 DOFs, capable of supporting the weight of a patient's arm and assisting the patient during specific exercises, adapting to the patient's capabilities, in a large $3 \mathrm{D}$ workspace ${ }^{[4]}$. Several researchers are still researching and developing novel design solutions for limb re- habilitation, including commercial devices ${ }^{[6]}$, exoskeletons such as reported, for example, in Refs. [7-10], or new kinematic architectures ${ }^{[11,12]}$ or cable driven parallel architectures as, for example, reported in Refs. [13-20]. The existing devices are often expansive, bulky, and complex to operate, requiring a specifically trained operator and dedicated facilities in specialized clinic environments. This can limit the spread of these solutions in the market especially when referring to less developed/low-income countries and/or in areas, which are not easily accessible.

This paper addresses the above-mentioned open problem by proposing a new design solution that can be easily portable also for home use. Other valuable features can be foreseen, for instance, in the possibility to keep social distancing while allowing rehabilitation treatments even during a pandemic spread. At the same time such device should provide performances comparable with the existing commercial design solutions and be suitable for a remote supervision by operators in clinics, who will adjust the treatment according to the patient needs. Given these main design requirements, a specific design procedure has been herewith outlined by focusing on the main steps of the mechanical design synthesis. This process included the development of specific kinematic, dynamics and FEM models and

\footnotetext{
*Corresponding author: Giuseppe Carbone

E-mail: giuseppe.carbone@unical.it
} 
numerical simulations. Attention has been given also to the outline of a specific operation architecture and sensory feedbacks. Preliminary experimental tests are reported to show the effectiveness and feasibility of the proposed design solution.

\section{Kinematic design}

A preliminary analysis was conducted to identify the characteristics of existing commercial devices for upper limb rehabilitation. A comparison has been made on basis of costs, workspace, and payload features. Results are summarized in Table 1. The comparison shows InMotion device has the solution having the simplest kinematic architecture, which, however, results in a very limited number of allowed planar motions, as based on only two Degrees of Freedom (DoFs). All the addressed commercial devices show a price close or higher than 100.000 US\$ with ArameoSpring being the cheapest product. Nevertheless, this corresponds to a significantly lower payload (close to half as compared tothe other devices in Table 1) while several motions cannot be actively controlled. All the addressed devices declare a payload capacity lower than $8 \mathrm{~kg}$ with a poor own device weigh/payload ratio and very heavy total weight, which significantly affects the portability. Most devices show a similar workspace size that is fitting with the average motion range of a human upper limb.

The analysis of existing devices identifies high costs and own weight as the most critical limitations that prevent the implementation of the existing devices for home use. Among the existing devices, ReoGo results to be the most promising solution as it is the cheapest and lightest solution. It has a simple kinematic architecture based on a spherical joint that is allowing three rotations about the center of the spherical joint. This device demonstrates a user-friendly operation with the patient sitting on a standard chair. The operation of this device is quite simple as it is like a car gear lever. ReoGo allows a maximum payload of $5 \mathrm{~kg}$, which is an average value among the other commercial devices. Based on the potentials of ReoGo, further studies and clinical tests have been made with a real ReoGo device, which authors have tested as courtesy of ANMIC rehabilitation clinic in Crotone, Italy, Fig. 1. After several experimental tests and analysis on ReoGo this commercial product has been found still too bulky and expansive to be considered for a home use. Moreover, the number of active degrees of freedom and motion capabilities result as being insufficient for a full rehabilitation treatment. The above aspects give the motivation of this work towards the design of a novel robotic device aiming at overcoming the limitations of ReoGo device with a more compact, lightweight, and cheap design while keeping a workspace and payload performance as being comparable with the ReoGo device. It is to note that the proposed device, similarly to ReoGo device, is not intended to restrain the movements of the shoulder elbow or wrist, since the aim of the treatment is to allow a neurological rehabilitation.

However, external constraints can be added to treat a single joint. Furthermore, the proposed device can be attached to the hand or to different body parts such for example the elbow to achieve the rehabilitation or exercising of specific muscles or joints. Even two devices can be used at same time, for example, for mirroring exercises at left and right limb. Accordingly, a design procedure has been defined for achieving the above-mentioned features with a new design solution.

The proposed design procedure has been carried out according to the following main steps:

(1) Topology search;

(2) Dimensional synthesis;

(3) Motor and gearbox selection as based on dynamic simulations;

(4) Mechanical design of main link elements via FEM optimization;

(5) End-effector and sensor feedback architecture;

(6) Control architecture and user-interface;

(7) First experimental validation.

\section{Kinematic synthesis}

A topology search has been carried out by considering workspace shapes and dimensionless sizes. The main search criteria have been to match or exceed the workspace size/shape, payload, and motion capabilities of ReoGo while keeping a comparable or possibly smaller footprint. The topology search particularly focused at parallel kinematics architectures as they demonstrate high payload to own weight ratios, high workspace to own size ratios (compactness), as 
Table 1 Comparison of different robotic solutions

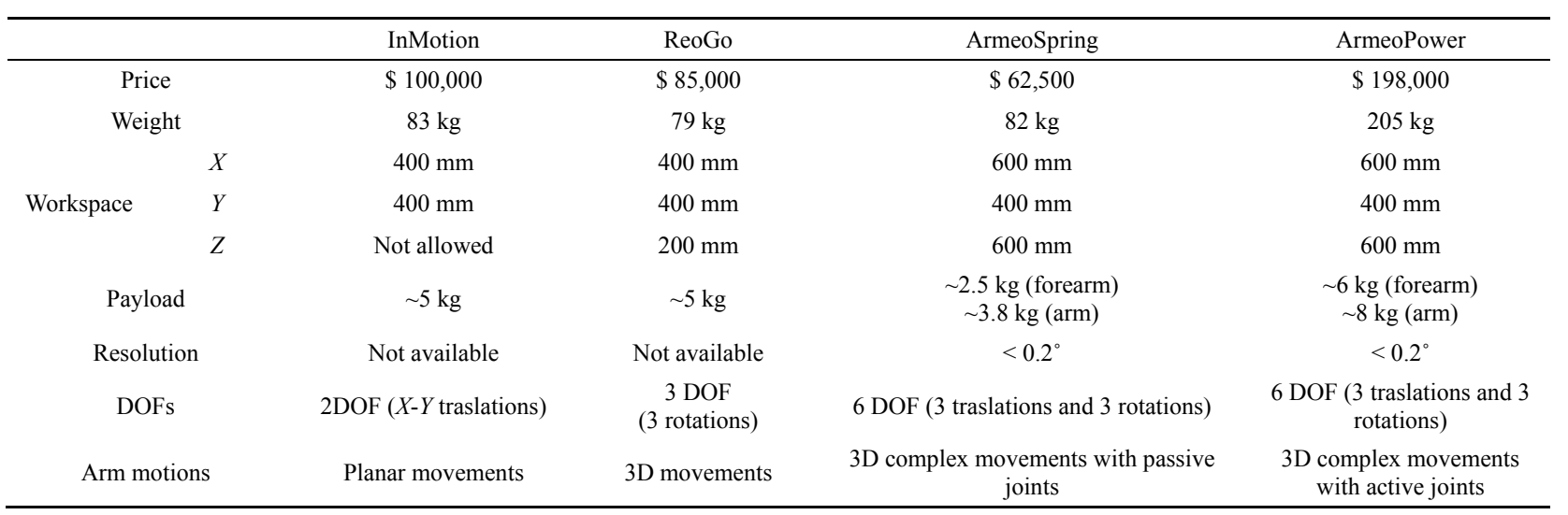

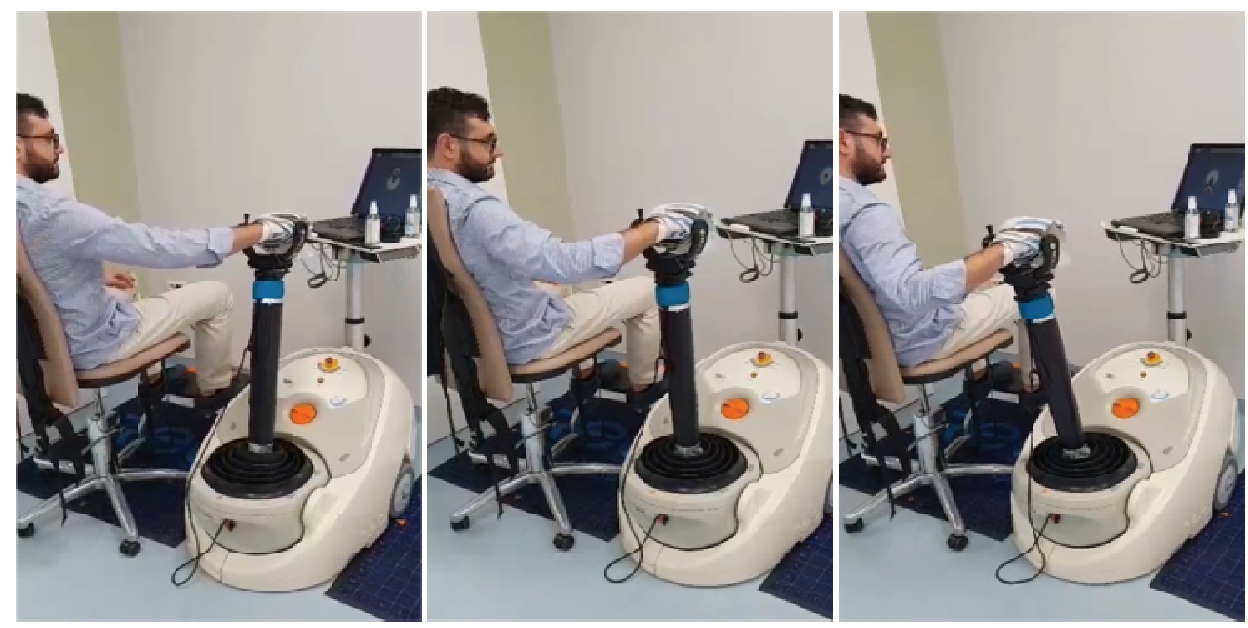

Fig. 1 First author testing ReoGo at ANMIC rehabilitation clinic in Crotone, Italy.

well as good dynamic performance with limited moving masses. The topology search considered several well-known parallel kinematics architectures such as those reported in Figs. 2 and 3. At this stage, the topology search was focused mostly on kinematic aspects by considering the shape and size of the achievable workspace as compared with the desired workspace of the ReoGo device in Fig. 1. In addition to the above-mentioned main search criteria on workspace, additional design constraints have been given for avoiding linear joints/actuators as well as keeping the robot size sufficiently compact to be placed on a common desk at home. The comparison of the numerically simulated workspaces in the examples of Figs. 1 and 2 shows the characteristics and complexity of the workspace shapes that can be achieved by a Delta-like with prismatic actuators and an R-Cube-like parallel archi- tecture, respectively. A first round of numerical simulations aimed at identifying and comparing the own sizes of a robot architecture to fulfill the desired workspace of the ReoGo device in Fig. 1. This process has allowed to identify a Delta-like parallel architecture with only revolute joints as the most promising for obtaining a compact solution with a potential high payload to own weight ratio ${ }^{[21]}$. This topology choice was based also on practical aspects including aspects of easy manufacturability and large use of low-cost off-the-shelf components. The final chosen topology is reported in Fig. 4. Fig. 4 also reports a numerical simulation of the workspace for the proposed Delta-like parallel architecture. After this first step, kinematic equations have been established and numerically solved in Matlab environment for performing a size synthesis.

Theoretical study of preferred solution starts from 

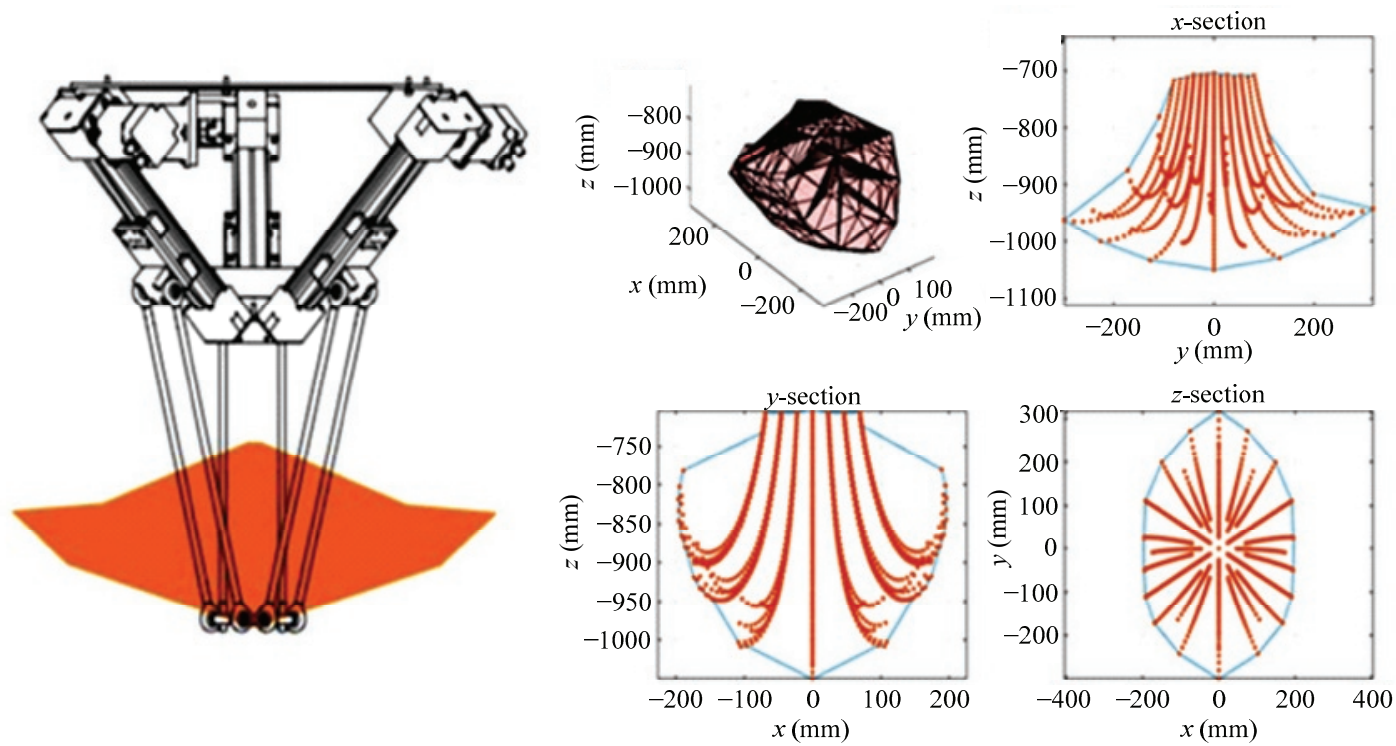

Fig. 2 Linear Delta robot's configuration and its workspace.
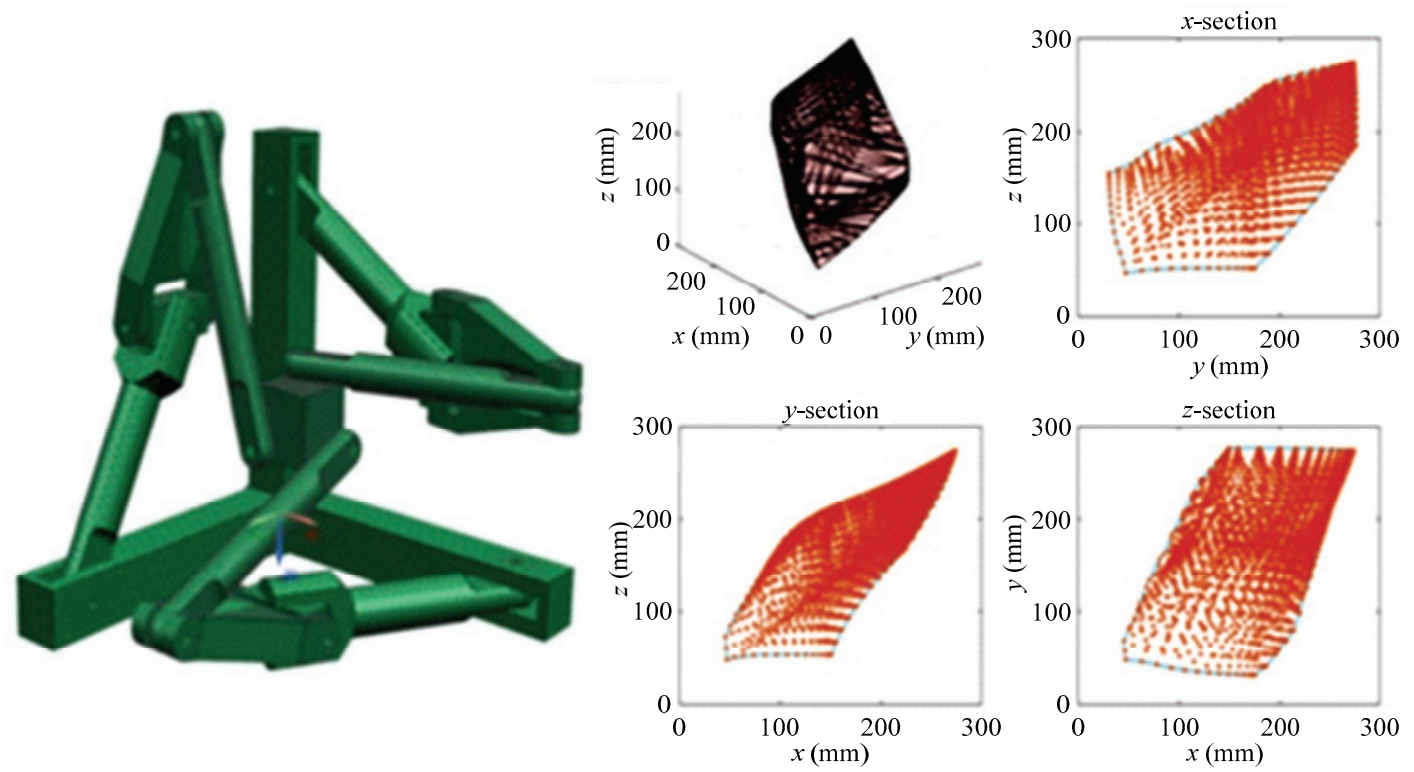

Fig. 3 R-Cube robot and its workspace.

establishing the kinematic equations, by referring to the schemes shown in Fig. 4, as reported in the following formulation. In particular, Fig. 4a shows a top view of kinematic scheme of the proposed Delta-like architecture. Fig. $4 \mathrm{~b}$ reports a detail of the proposed geometric approach where one can identify the position of the origin $\mathrm{O}$ of the movable plate (end-effector) relative to a fixed reference frame that attached to point Eo on the fixed platform by using a geometric relationship given by closing the three vector loops $\boldsymbol{E}_{\boldsymbol{o}} \boldsymbol{E}_{\boldsymbol{i}}+\boldsymbol{E}_{\boldsymbol{i}} \boldsymbol{J}_{\boldsymbol{i}}+\boldsymbol{J}_{\boldsymbol{i}} \boldsymbol{F}_{\boldsymbol{i}}+\boldsymbol{F}_{\boldsymbol{i}} \boldsymbol{O}$ $=\boldsymbol{P}$ with $i=1,2,3$, respectively. The three vector loops can be identified in the 3D kinematic scheme in Fig. 4c. Note that the movable reference frame $O X Y Z$ is rigidly linked to the movable plate (end-effector), whose position and orientation changes due to changes in the input variables $\theta_{1}, \theta_{2}, \theta_{3}$, which produce changes the position of the points $J_{1}, J_{2}, J_{3}$ versus time. In particular, Fig. $4 \mathrm{~d}$ reports an arbitrary configuration at time equal to $t^{\prime}$ 


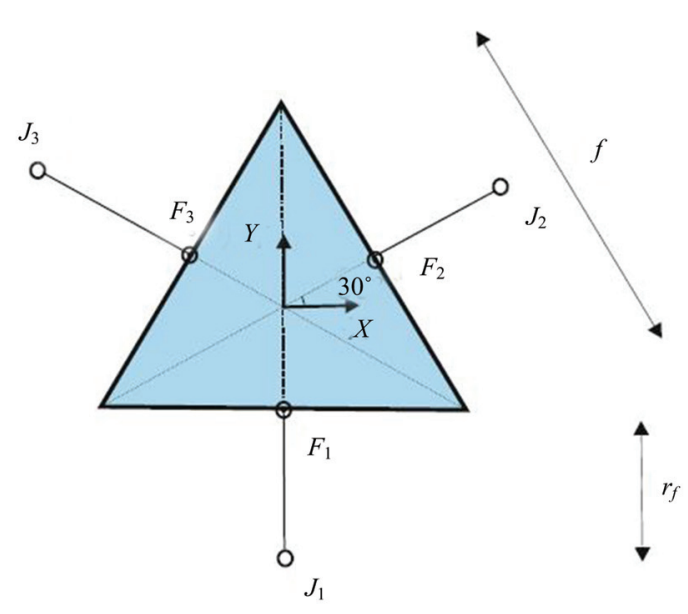

(a)

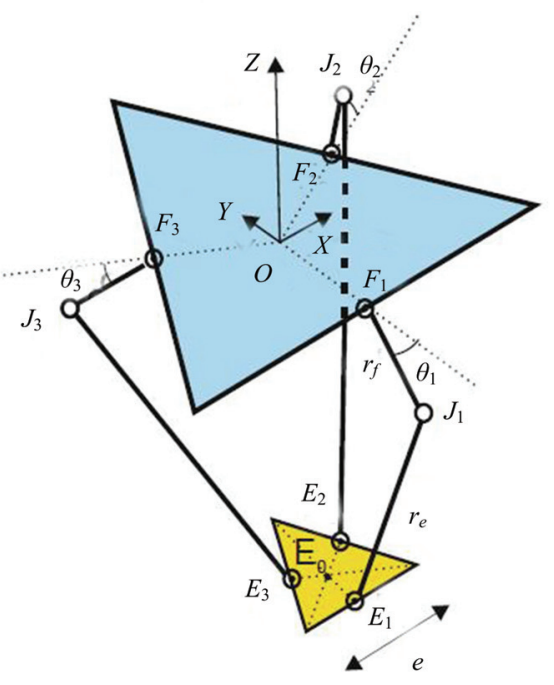

(c)

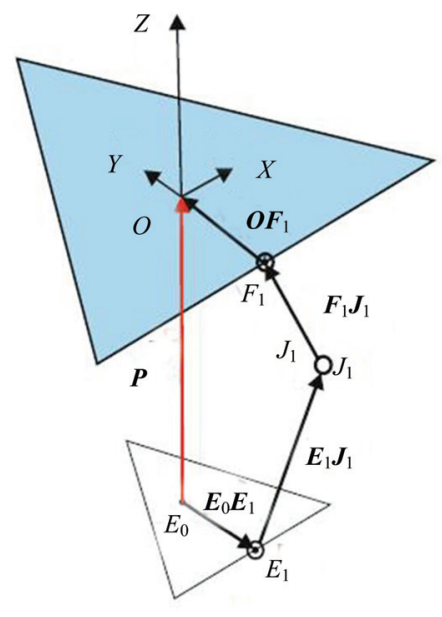

(b)

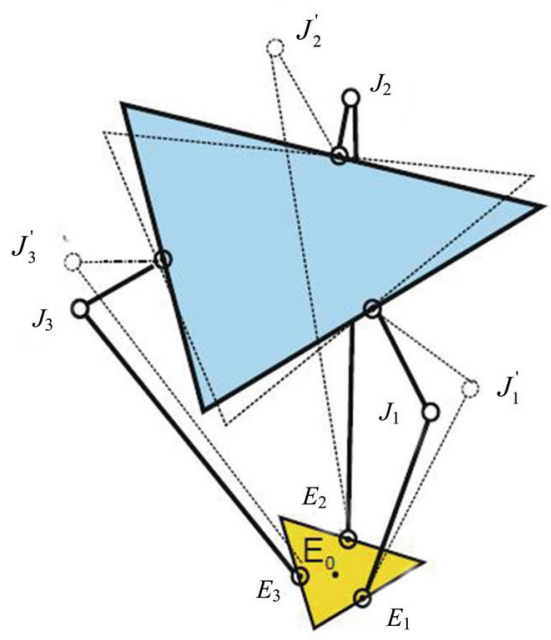

(d)

Fig. 4 A kinematic schemes of the proposed Delta-like architecture: (a) a top view of the mobile platform (end-effector); (b) a detail of the proposed geometric approach; (c) a 3D kinematic scheme; (d) a scheme highlighting a small motion of the end-effector (points $J_{1}, J_{2}, J_{3}$ move to points $J_{1}^{\prime}, J_{2}^{\prime}, J_{3}^{\prime}$, respectively).

where the position of the above-mentioned points becomes $J_{1}^{\prime}, J_{2}^{\prime}, J_{3}^{\prime}$, respectively.

Given the proposed geometric approach, referring to the schemes in Fig. 4, one can write:

$$
\begin{gathered}
\left|\boldsymbol{O} \boldsymbol{F}_{1}\right|=\left|\boldsymbol{O} \boldsymbol{F}_{2}\right|=\left|\boldsymbol{O} \boldsymbol{F}_{3}\right|=\frac{f}{2} \tan \left(\frac{\pi}{6}\right), \\
\left|\boldsymbol{J}_{1} \boldsymbol{J}_{1}^{\prime}\right|=\left|\boldsymbol{J}_{2} \boldsymbol{J}_{2}^{\prime}\right|=\left|\boldsymbol{J}_{3} \boldsymbol{J}_{3}^{\prime}\right|=\frac{e}{2} \tan \left(\frac{\pi}{6}\right), \\
\left|\boldsymbol{F}_{1} \boldsymbol{J}_{1}\right|=r_{f} \cos \left(\theta_{1}\right),
\end{gathered}
$$

$$
\begin{aligned}
& \left|\boldsymbol{F}_{2} \boldsymbol{J}_{2}\right|=r_{f} \cos \left(\theta_{2}\right), \\
& \left|\boldsymbol{F}_{3} \boldsymbol{J}_{3}\right|=r_{f} \cos \left(\theta_{3}\right),
\end{aligned}
$$

where $\theta_{1}, \theta_{2}, \theta_{3}$ define the orientations of the segments given by vectors $\boldsymbol{F}_{1} \boldsymbol{J}_{1}, \boldsymbol{F}_{2} \boldsymbol{J}_{2}, \boldsymbol{F}_{3} \boldsymbol{J}_{3}$, respectively; The operator || stands for absolute value.

From the Eqs. (1) to (5) it is possible to obtain the position of the rotation centers of the arms as:

$$
J_{1}^{\prime}=\left\{0 ;-\frac{(f-e)}{2} \tan \left(\frac{\pi}{6}\right)-r_{f} \cos \left(\theta_{1}\right) ;-r_{f} \sin \left(\theta_{1}\right)\right\},
$$




$$
\begin{aligned}
& J_{2}^{\prime}=\left\{\left[(f-e) / 2 \sqrt{3}+r_{f} \cos \left(\theta_{2}\right)\right] \cos (30) ;\left[(f-e) / 2 \sqrt{3}+r_{f} \cos \left(\theta_{2}\right)\right] \sin \left(\frac{\pi}{6}\right) ;-r_{f} \sin \left(\theta_{2}\right)\right\}, \\
& J_{3}^{\prime}=\left\{-\left[(f-e) / 2 \sqrt{3}+r_{f} \cos \left(\theta_{3}\right)\right] \cos \left(\frac{\pi}{6}\right) ;\left[(f-e) / 2 \sqrt{3}+r_{f} \cos \left(\theta_{3}\right)\right] \sin \left(\frac{\pi}{6}\right) ;-r_{f} \sin \left(\theta_{3}\right)\right\} .
\end{aligned}
$$

Once these points have been defined, one can define three spheres, which have as their center the points $J_{1}^{\prime}, J_{2}^{\prime}, J_{3}^{\prime}$, whose coordinates $\left(x_{1}, y_{1}, z_{1}\right)\left(x_{2}, y_{2}, z_{2}\right)$ and $\left(x_{3}, y_{3}, z_{3}\right)$ need to keep a distance from the end-effector as equal to $r_{e}$. Accordingly, the following three equations can be written as:

$$
\left\{\begin{array}{l}
x^{2}+\left(y-y_{1}\right)^{2}+\left(z-z_{1}\right)^{2}=r_{e}^{2} \\
\left(x-x_{2}\right)^{2}+\left(y-y_{2}\right)^{2}+\left(z-z_{2}\right)^{2}=r_{e}^{2} . \\
\left(x-x_{3}\right)^{2}+\left(y-y_{3}\right)^{2}+\left(z-z_{3}\right)^{2}=r_{e}^{2}
\end{array}\right.
$$

The Eq. (9) is a system of three equations where the values of end-effector's positions $(x, y, x)$ can be calculated as function of the input variables $\theta_{1}, \theta_{2}, \theta_{3}$. Accordingly, Eq. (9) have been implemented in Matlab environment with three nested for loops allowing to span the input variables $\theta_{1}, \theta_{2}, \theta_{3}$ from their lower bound to their upper bound with a desired angular step size. This numerical algorithm allows to numerically calculate as output a cloud of reachable values of the end-effector's position $(x, y, x)$. Number of calculated points increases by reducing the angular step size resulting in a denser cloud of points. As tradeoff any reduction of the angular step size results in an increase of the computational costs. Note that the calculated cloud of end-effector's position $(x, y, x)$ points is needed for a size synthesis design of the proposed device one can identify a reasonable practical tradeoff value for the angular step size for this specific case as $5^{\circ}$. Note that the calculations done through Eq. (9) allow to calculate the envelope of the workspace. A first iteration can calculate the upper bound as intersection of three spheres, whose radiuses correspond to the maximum reaches. A further iteration can use the same approach for calculating the inner envelope of the workspace as intersection of three spheres, whose radiuses correspond to the minimum reaches. The above-mentioned iterations are made within for loops where the input variables $\theta_{1}, \theta_{2}, \theta_{3}$ are increased incrementally from the lower bound to the upper bound with increments of $5^{\circ}$ to span the whole workspace. The ob- tained output consists of the reached pose of the end-effector ( $x, y, z$ coordinates) as function of a specific set of input variables $\theta_{1}, \theta_{2}, \theta_{3}$. The whole iterations can be achieved in less than 1 second of computational time in a standard laptop computer equipped with Matlab environment. One should note that the overall workspace can be also analytically calculated as intersection of three torus consisting of the portions of the spheres obtained with a radius ranging from the minimum to the maximum reach.

Results of the size synthesis process have been the main sizes of the proposed Delta-like architecture. Accordingly, main obtained sizes are reported in Table 2. An algorithm has been implemented in Matlab to generate the workspace, whose output is shown in Fig. 5. The sizes in Table 2 allow to obtain a workspace, which fits the prescribed operation workspace and slightly exceed ReoGo workspace. Further design steps have been made to slightly exceed ReoGo performance in terms of payload, and motion capabilities. Iterative design steps have been made also to achieve a compact overall robot size and to verify the footprint size, which fits on a common desk at home. It is to note that medical protocols do not require to operate the human upper limb through its whole workspace, since the aim of the treatment is not to exercise the muscles and joint. Instead, aim is to achieve a neurological rehabilitation consisting of regaining neurological control of the upper limb. In this sense ReoGo is a commercial standard with established clinical protocols. For this reason, the target of this work is to replicate at home the clinical protocols that have been established for ReoGo, which, however, can only be used in clinics.

Table 2 Main obtained sizes as referring to the scheme in Fig.1

\begin{tabular}{ccccc}
\hline & Obtained design parameters & & $\begin{array}{c}\text { Obtained motion per- } \\
\text { formance }\end{array}$ \\
\hline$f$ & $r_{f}$ & $r_{e}$ & $e$ & $\begin{array}{c}\text { Achievable vertical } \\
\text { motion ranging from } \\
444 \mathrm{~mm} \text { to } 577 \mathrm{~mm}\end{array}$ \\
$350 \mathrm{~mm}$ & $130 \mathrm{~mm}$ & $300 \mathrm{~mm}$ & $90 \mathrm{~mm}$ & \begin{tabular}{c}
447 \\
\hline
\end{tabular}
\end{tabular}



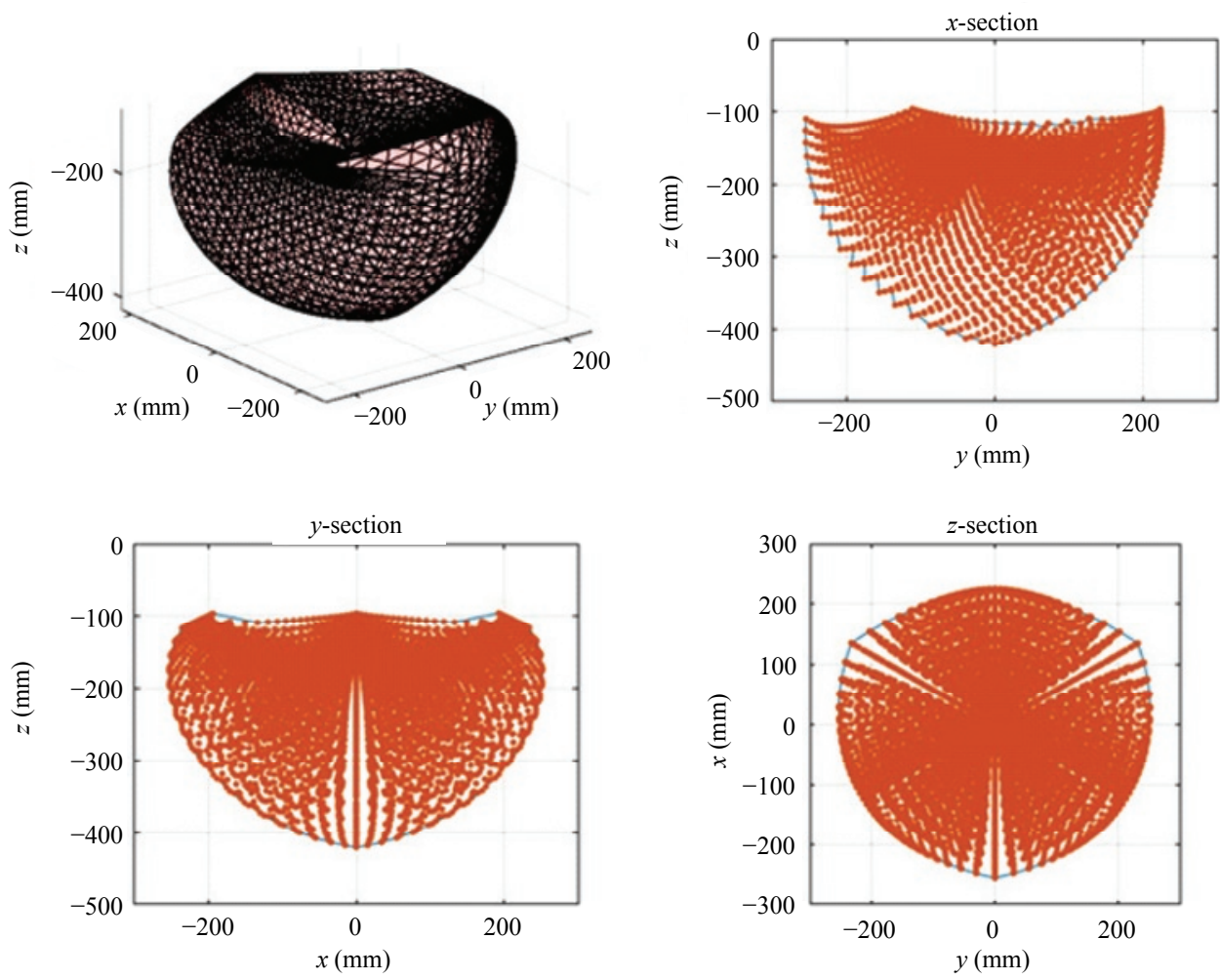

Fig. 5 Plots of the workspace for the proposed device (sizes are given in $\mathrm{mm}$ ).

\section{Mechanical design}

Following the kinematic synthesis, the design process has been focusing on motor and gearbox selection as based on kinematic and dynamic simulations. For the purpose, a specific 3D CAD model has been developed in Solidworks environment, as reported in Fig. 6. This CAD model has been used for estimating all the masses and inertias as well as for implementing motion simulations by means of the Solidworks toolbox motion. Simulations allowed to verify the desired motion ranges and payloads as well as to properly size the motors, gearboxes, and joints. Ergonomics was carefully considered at all design stages, especially, for the human-robot interface, which can be easily adapted and personalized to a user with smooth and soft surfaces.

Dynamic simulations have been carried out with two approaches. A first approach consists of establishing a simplified analytical dynamic model, which has been developed as based on the principle of virtual powers ${ }^{[22]}$. The related solving algorithm has been developed in a symbolic solver environment (Mathematica software). This analytical model requires as input the position of

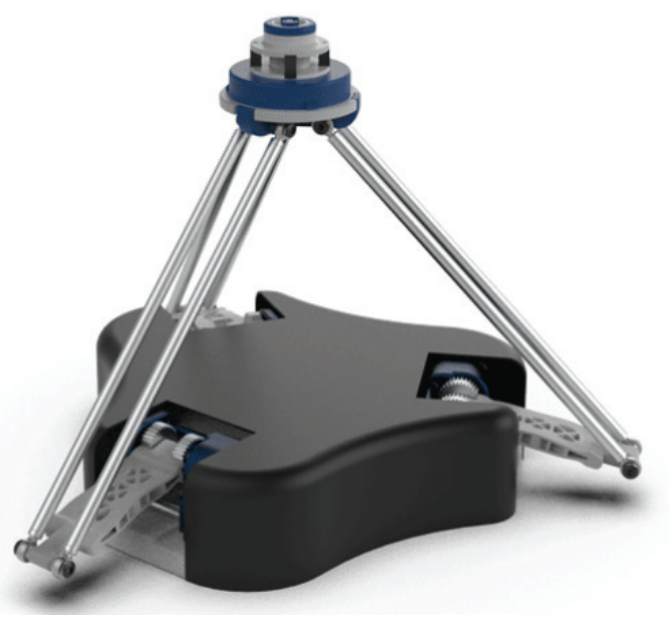

Fig. 6 3D CAD rendered model of the proposed robotic device.

the centers of mass versus time. For the purpose, a law of motion has been imposed for joints to fulfill a specific vertical motion of the end-effector along the $Z$-axis. The motion laws have been obtained starting from the analytical formulation of direct kinematics as reported for example in Ref. [21]. Fig. 7 shows the calculated paths that are performed by centers of masses, for example, when the manipulator moves along a prescribed vertical 


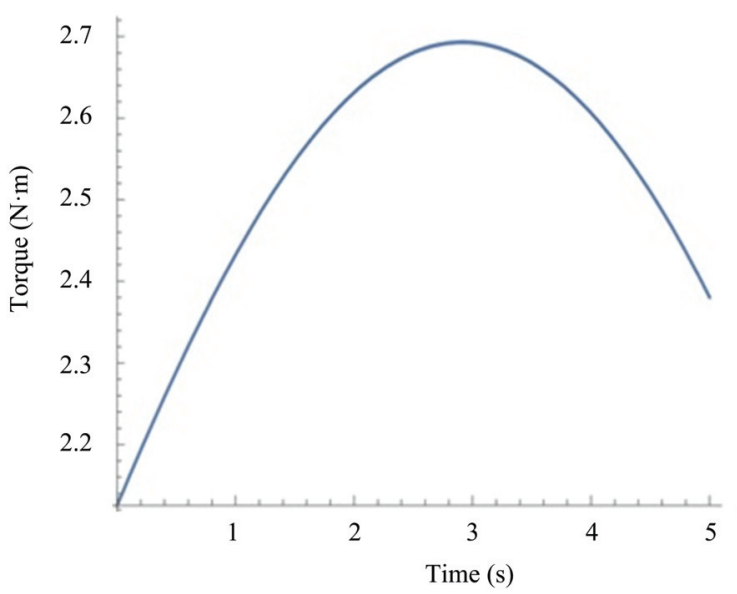

Fig. 7 Simulation results for a prescribed vertical motion of the end-effector along the $Z$-axis in terms of required motor torque $(\mathrm{N} \cdot \mathrm{m})$ versus time $(\mathrm{s})$.

motion of the end-effector along the $Z$-axis. Paths of the center of masses can be computed for any desired motion of the manipulator. The main output of the solution of the analytical dynamic model consists of the required input motor torques. For example, Fig. 7 shows the motor torque versus time for the prescribed vertical motion of the end-effector along the $Z$-axis. Given the symmetry of this prescribed motion all motors require the same torque. The maximum torque during this movement is about $2.7 \mathrm{~N} \cdot \mathrm{m}$. Also, other motions have been considered. All of them have been found to require a lower maximum input torque than the above-mentioned general case.

To determine the required motor torque in a preliminary way, the principle of virtual powers was used to equal the input power $\left(\right.$ Power $\left._{\text {in }}\right)$ and the output power $\left(\right.$ Power $\left._{\text {out }}\right)$. In fact, if one assumes any dissipative effect as negligible, one can write

$$
\text { Power }_{\text {in }}=\text { Power }_{\text {out }} \text {. }
$$

If one refers to a symmetric configuration, all motors contribute equally to the input power. Accordingly, Eq. (10) yields to:

$$
T_{\text {in }}(t) \omega_{\text {in }}(t)=\frac{1}{3}\left(M_{\text {arm }} g\right) V_{\text {out }}(t),
$$

where $T_{\text {in }}$ stands for the input torque, $\omega_{\text {in }}$ stands for the input angular velocity, $M_{\text {arm }}$ stands for the mass of an arm lumped in its center of mass, $V_{\text {out }}$ stands for the absolute velocity of the end-effector. Accordingly, it is possible to obtain the torque values through the following:

$$
T_{\text {in }}(t)=\frac{1}{3}\left(M_{\text {arm }} g\right) \frac{V_{\text {out }}(t)}{\omega_{\text {in }}(t)} .
$$

As next step, further simulations have been carried out by developing a specific model within MSC ADAMS environment. This allowed a more accurate dynamic modelling of the proposed device as well as simulation of several operation conditions. This MSC ADAMS simulation has been achieved by considering a simplified 3D multibody model. Joints have been carefully selected to avoid redundant constraints issues. A GSTIFF numerical solver has been selected. Friction has been considered as negligible since all joints use ball bearings or low friction spherical joints. In particular, the device has been simulated by performing a general trajectory covering all the allowed motion directions with maximum reaches within the allowed workspace. The whole simulation has been set to last 30 seconds while following the motions listed in Table 3.

The proposed MSC.ADAMS simulations allow to obtain several information on the dynamic behavior of the proposed device. Some examples of the obtained results are reported in Fig. 8. All the simulations have confirmed the results of the theoretical model in terms of maximum required torque as equal to $2.7 \mathrm{~N} \cdot \mathrm{m}$ when a maximum patient force $5 \mathrm{~kg}$ is applied to the end-effector.

Note that the limb own weight is also considered in the proposed model with a conservative additional payload of $5 \mathrm{~kg}$. This leads to a total of $10 \mathrm{~kg}$ applied to the end-effector in the worst-case scenario.

This maximum payload has been defined by considering the same maximum allowed payload of the commercial ReoGo as well as it is providing a safety factor equal to about 2 when compared with the average

Table 3 Main data of the simulated motions in MSC.ADAMS

\begin{tabular}{cc}
\hline Time interval (s) & Output motion of the end-effector \\
\hline $0 / 5$ & Initial configuration/ $+200 \mathrm{~mm}$ along $X$-axis \\
$5.1 / 10$ & $+200 \mathrm{~mm}$ along $X$-axis/-200mm along $X$-axis \\
$10.1 / 15$ & $-200 \mathrm{~mm}$ along $X$-axis $/+200 \mathrm{~mm}$ along $Z$-axis \\
$15.1 / 20$ & $+200 \mathrm{~mm}$ along $Z$-axis $/-200 \mathrm{~mm}$ along $Z$-axis \\
$20.1 / 25$ & $-200 \mathrm{~mm}$ along $Z$-axis $/+30 \mathrm{~mm}$ along $Y$-axis \\
$25.1 / 30$ & $+160 \mathrm{~mm}$ along $Y$-axis $/+380 \mathrm{~mm}$ along $Y$-axis \\
\hline
\end{tabular}



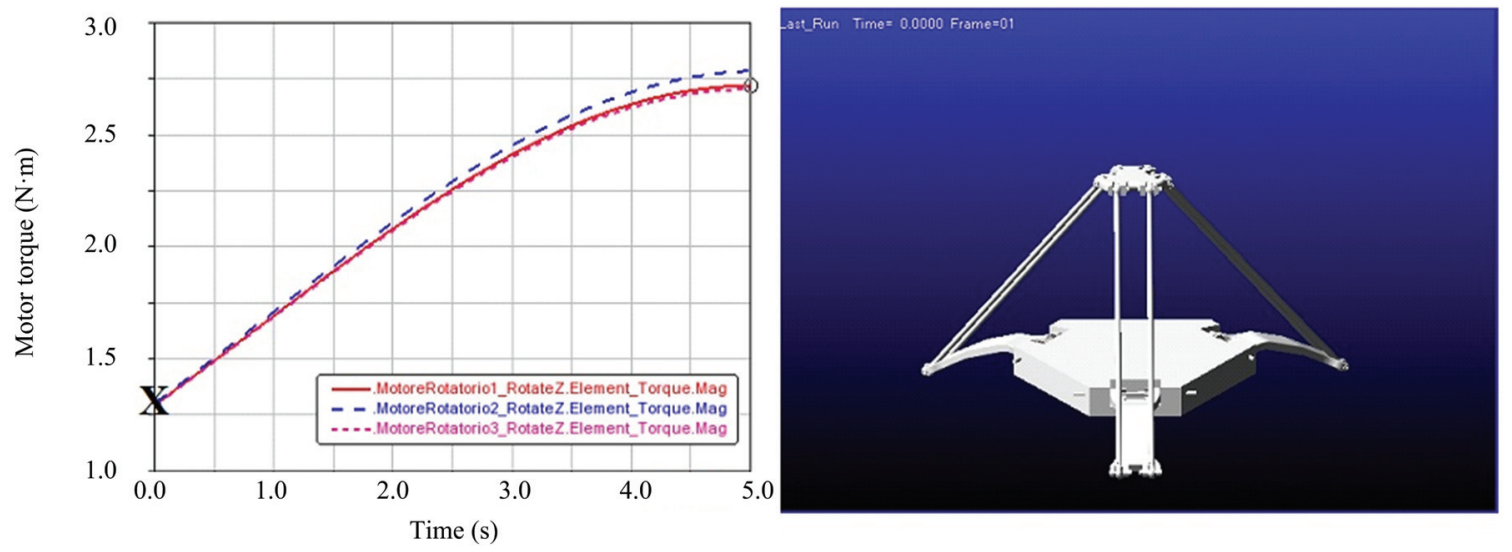

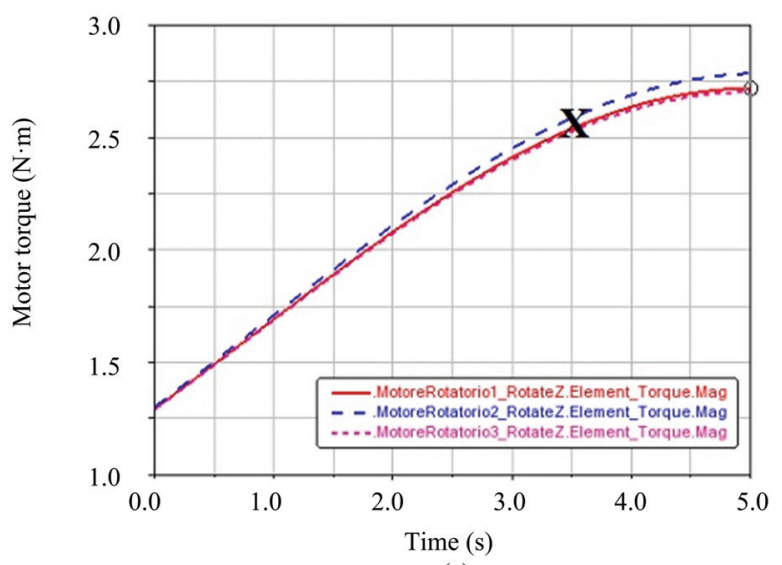

(a)

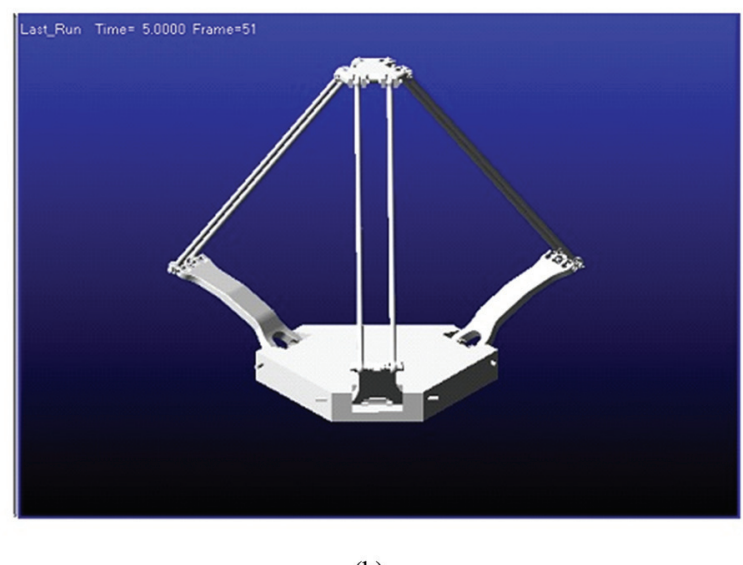

(b)

Fig. 8 Simulation results obtained with MSC.ADAMS: (a) simulated motor's torques expressed in (N·m) versus time (s); (b) robot configurations corresponding to the values indicated in the simulation plots at the values marked with $\mathrm{X}$.

human upper limb weight as reported for example in Refs. [23-25].

Nema-17 stepper motors have been selected as input motors as they can provide a compact, cheap and easy to operate solution. However, these motors are too fast, and they generate a too low input torque as compared with the obtained simulated values. Accordingly, these motors require a gearbox for fulfilling the required speed and torque. For the purpose, given the compactness requirements, it has been decided to design a specific epicyclical gearbox. After a careful design process, the adopted solution has three gears with 10,17 and 44 teeth, respectively.

In order to allow the gears to mesh, the space between the gear teeth of each gear (the pitch p) must be equal. The pitch $\mathrm{p}$ of a gear indicates the size of the gear tooth. Dividing the pitch by the constant value $\pi$ one obtains the module $m=p / \pi$. In standard gears with modular design, the tooth height is equal to $2.25 \mathrm{~m}$. One should not that a small $\mathrm{m}$ results in smallteeth, which can allow a compact design. On the other side, small module values can generate transmission problems, which are discussed in detail for example in Ref. [22]. After an iterative design process, the gear module of all gears has been defined as equal to 0.9 allowing a sufficiently compact design and fulfilling the required torque transmission without motion interference, as based on the design process also discussed in Ref. [22].

To size the other robot components, we started from the data obtained from the multibody simulations in MSC.ADAMS environment. In particular, one has to consider the loading conditions that are acting on the arms, which are reported in the Fig. 9. The main forces acting are the flexion and the torsion. The geometry was simulated in Abaqus FEM software, as shown in Fig. 10. The model has been implemented by considering a 


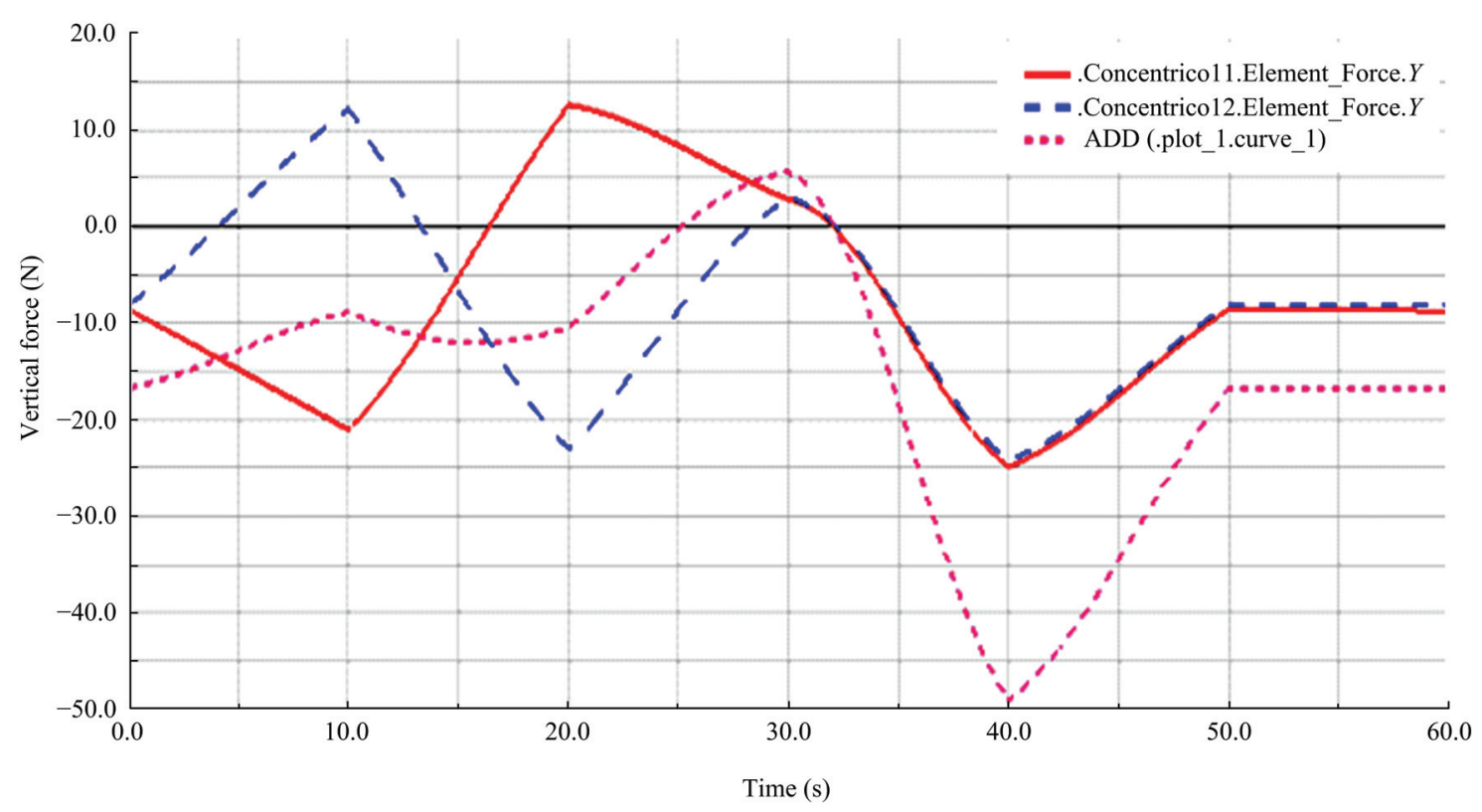

Fig. 9 Simulated vertical force $(\mathrm{N})$ versus time (s) on the center of the spherical joint at the movable plate as o from the model in Fig. 8.
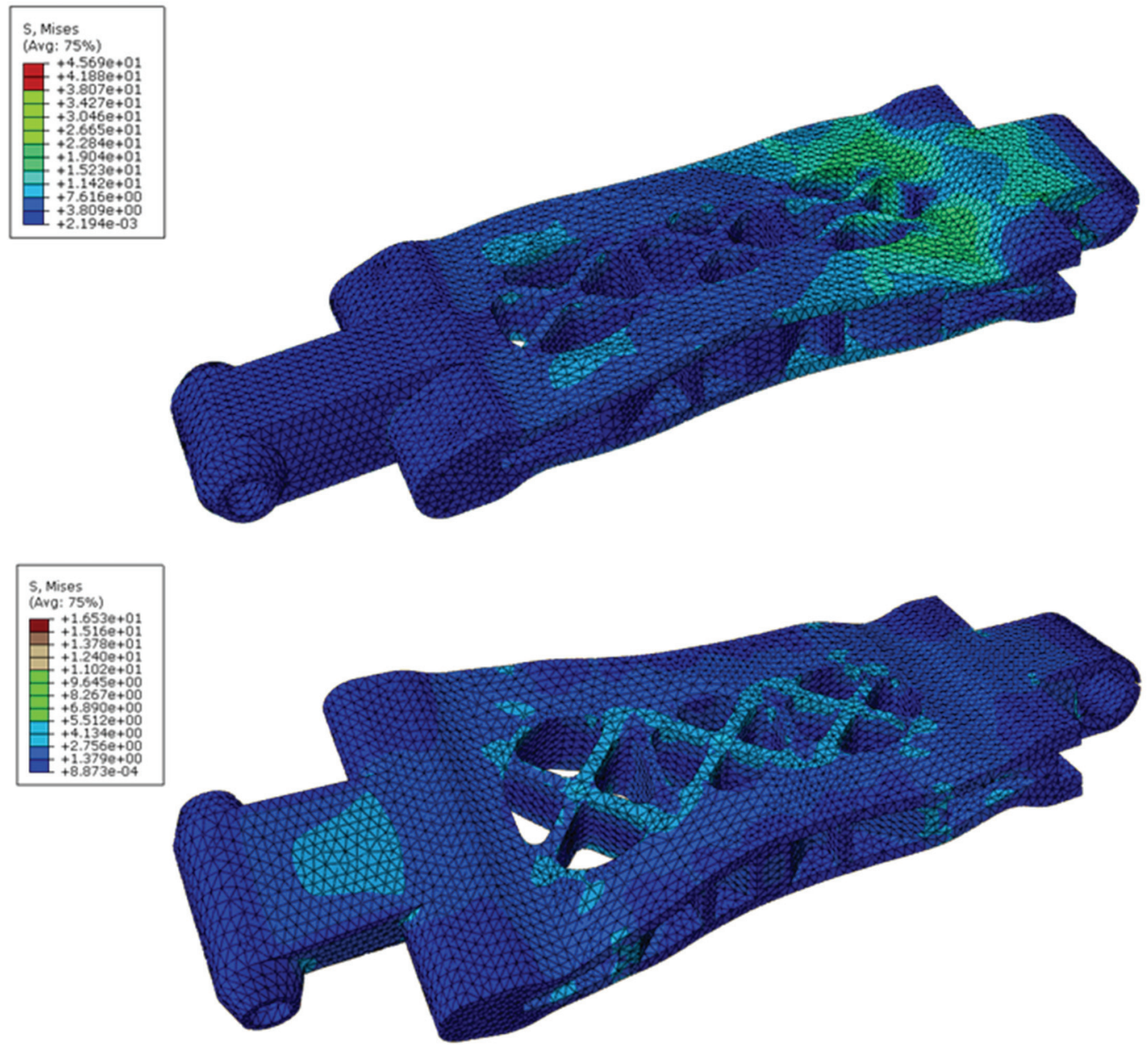

Fig. 10 The obtained FEM analysis of the proposed robot arms in Abaqus FEM software where dark blue to green colors stand for safe stiff material behaviors. 
standard PLA material, which is the plastic commonly used for 3D printing. Accordingly, the obtained FEM results will validate the effectiveness of producing a first prototype with a conventional 3D printing technique. It is to note that the Abaqus FEM model has been also used for optimizing the arm shapes for achieving an optimal tradeoff between stiffness and arm weight. The final arm shapes are shown in Fig. 10.

\section{Control architecture and user interface}

After completing the mechanical design of the proposed robot, attention has been focused on the robot end-effector and sensory feedback. The end-effector is the terminal part of the robot, which has the task of interacting with the patient with the machinery. It represents the perceptive part of the machine as it can be equipped with sensors at the human-robot interface. A specific end-effector design is proposed. This effector is mostly an ergonomic base for housing the hand. The straps can be also included for holding and fixing the hand on the device. Ideal set-up would include a six-axis force/torque sensor. However, these devices are quite expansive and not easy to miniaturize. Accordingly, here we propose a low-cost solution with six miniaturized lever switches, which can detect and map the patient direction of motion along the three translational axes $X$-, $Y$-, $Z$ - (positive or negative directions). In this way the device can understand in which direction the patient's hand is pushing and can decide whether to support the movement, guide or contrast it as based on the training to be performed. The whole device weights less than $1 \mathrm{~kg}$ excluding motors with the main sizes that are reported in Table 2. The end-effector weights about $300 \mathrm{~g}$. The estimated positioning accuracy is lower than $1 \mathrm{~mm}$. This specific set up is currently covered by aninternational patent application.

The above-mentioned sensor feedback is integrated in a simple Arduino-based control architecture. Accordingly, the robot manages to interpret the patient's motor intentions using the sensor feedbacks and moves the robot using the inverse kinematics implemented in the control software. The control architecture is based on Arduino UNO controller as a simple and reliable laboratory set up. Other main components of the proposed control architecture are: three stepper motors with re- lated drivers, an AC/DC stabilized IO 110/220 V OU $12 \mathrm{~V}-10$ A power supply, limit range safety switches and an LCS screen. Communication with a PC is achieved through a serial port, exchanging I/O data on the same channel. Arduino's digital PINs are used to control the stepper motors and to navigate into menus using three buttons, while an Arduino's analog PIN is used for sensors, by using a mapping method on a single analog PIN. In this way, it is possible to register the motion interaction with the patient's hand.

A specific software has been implemented to achieve a simple user-friendly control strategy where the LCD screen and to buttons allow for accessing all the main robot functionalities including a rehabilitation/exercising operation mode, and a parameter setup operation mode. In the parameter set up mode it is possible to select the rehabilitation/exercising operation parameters including, for example, guiding mode/contrast mode/assistance mode, the motion speed, the percentage of motor assistance in guiding mode, or the percentage of motor resistance in contrast mode, movements can be also limited to a prescribed path or in random movements. As future work visual interactions can be implemented to increase the user interactions as well as specific, cloud based, remote monitoring features and additional sensory feedbacks (i.e. EMG sensors to detect muscle stimuli) also one can considered other rehabilitation motions or control strategies, as proposed for example in Refs. [26-28].

\section{Preliminary tests}

This section presents some preliminary experimental tests that have carried out to validate the main features of the prototype. For example, Fig. 11 shows the built prototype while performing the prescribed vertical motion of the end-effector along the $Z$-axis, which has been described in Fig. 11. The experimental tests have demonstrated the engineering feasibility of the proposed device and the fulfillment of all the design requirements described at section 2, exceeding ReoGo performance while operation on a common home desk. The user was able to comfortably use the device with no problem in all operation conditions. Adaptability to various user heights has been easily achieved by using a standard chair with adjustable height. 
Further, experimental tests have been carried out with objects of different weights until reaching the prescribed payload of $5 \mathrm{~kg}$. Several trajectories have been tested. For example, Fig. 12 shows a test for the prescribed motions along $X-, Y$-, $Z$ - axes of the end-effector while lifting a smartphone. These experiments have been carried out at three different input velocities. An IMU sensor on the end-effector has been used for measuring the acceleration data, as reported in Fig. 13. It is worth noting that high vibrations can be identified at slow speed at about $0.1 \mathrm{~cm} \cdot \mathrm{s}^{-1}$. This is mostly due to working principle of the stepper motors as well as by the prototype characteristics of the gearboxes. However, a proper smooth motion is achieved at nominal operation speed. The results of the above-mentioned preliminary experiments confirm that the device performs the same movements, motion ranges and payload of a ReoGo commercial device while it can be conveniently placed on a common desk at home. The overall costs are promising as currently being below a total of 1,000 Euros, for a 3D printed single prototype, including all components, machining, assembly, main electronic components. Its software has been also developed in-house with all the required main operation features. Cost reductions can be expected for mass productions. Further experimental tests with patients will be carried out in future to prove the feasibility of applying the proposed device within specific clinical protocols.

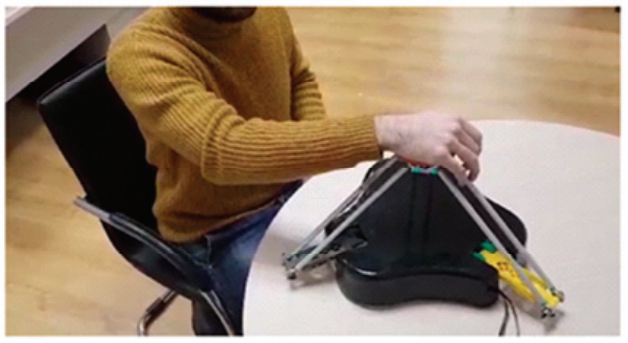

(a)

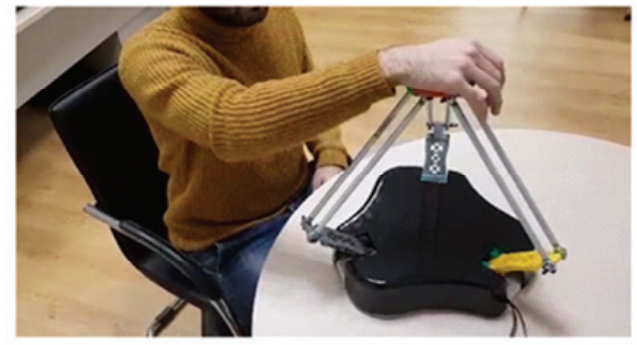

(b)

Fig. 11 Preliminary tests with a prototype: (a) minimum lift configuration; (b) maximum lift configuration.
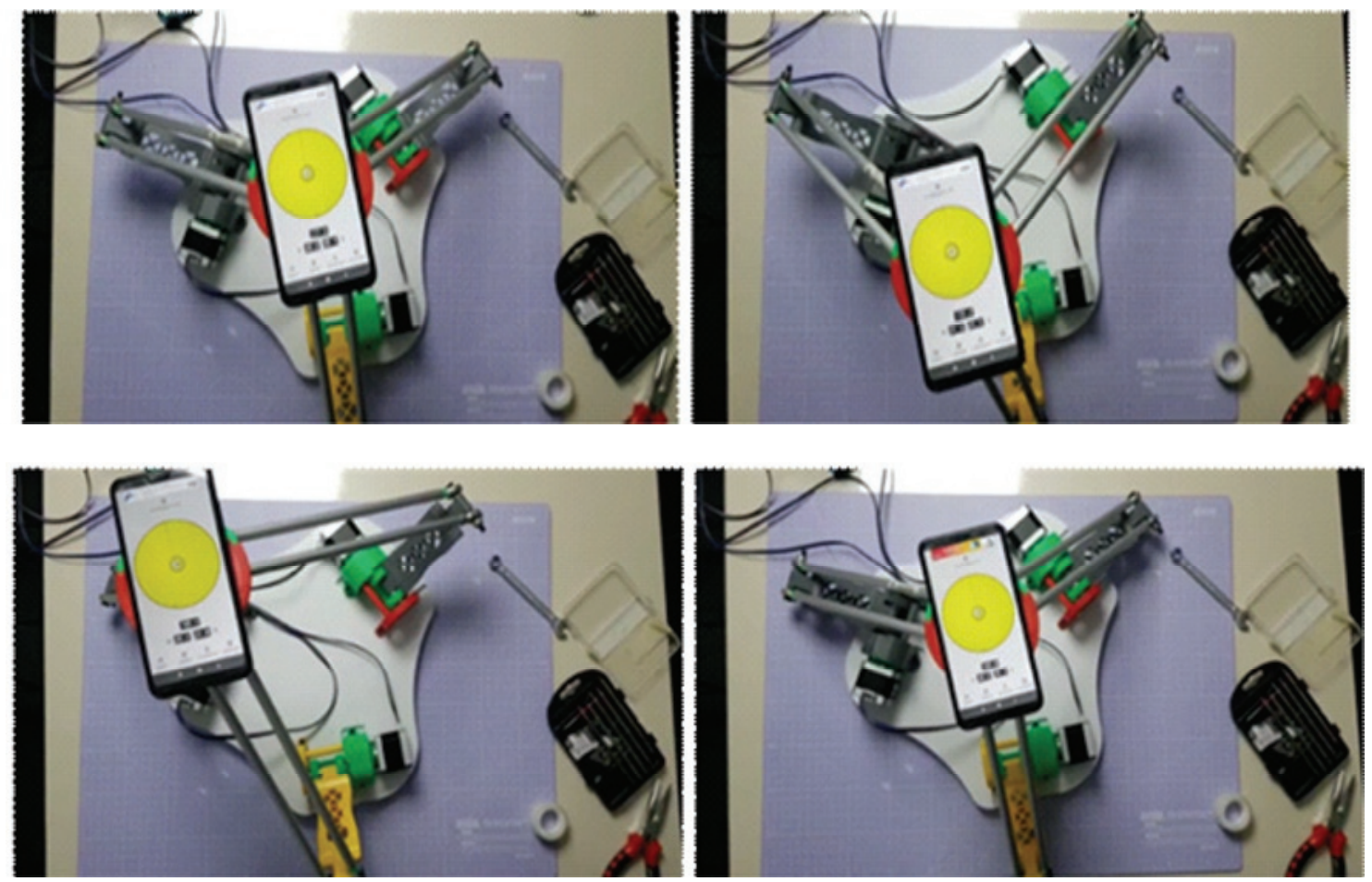

Fig. 12 A robot testing with a smart phone on its end-effector. 

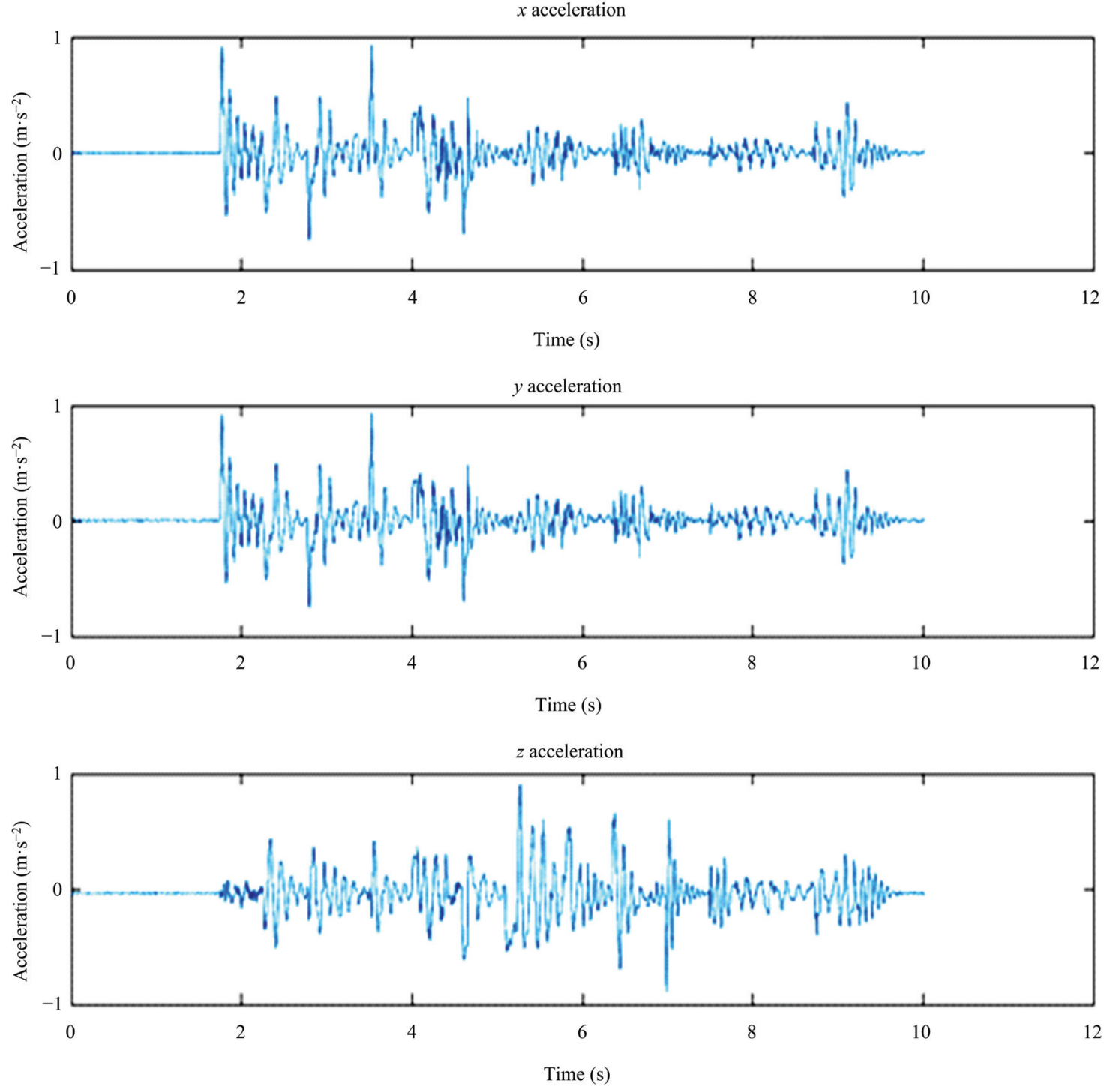

Fig. 13 Plots of the IMU data for the experiment in Fig. 8 at increasing speeds.

\section{Conclusion}

This paper outlines the design process for achieving a novel robotic device that is intended for upper limb rehabilitation. The proposed procedure includes a topology search. The proposed device has been designed as based on a Delta-like parallel architecture. Kinematic and dynamic simulations have been carried out for achieving the size synthesis of the main components, including a specific epicyclical gearbox. A prototype has been 3D printed and preliminarily validated. Experimental tests demonstrate the feasibility of the proposed concept to perform the same movements, motion ranges and payload of a ReoGo commercial device while it can be conveniently placed on a common desk at home. The overall performance of the designed device looks promising for allowing remote rehabilitation treatments at home even in low-income difficult-to-reach regions. Further experimental tests with patients will be carried out in future to prove the feasibility of applying the proposed device within specific clinical protocols.

Funding Open access funding provided by Università della Calabriawithin the CRUI-CARE Agreement.

Open Access This article is licensed under a Creative Commons Attribution 4.0 International License, which permits use, sharing, adaptation, distribution and re- 
production in any medium or format, as long as you give appropriate credit to the original author(s) and the source, provide a link to the Creative Commons licence, and indicate if changes were made.

The images or other third party material in this article are included in the article's Creative Commons licence, unless indicated otherwise in a credit line to the material. If material is not included in the article's Creative Commons licence and your intended use is not permitted by statutory regulation or exceeds the permitted use, you will need to obtain permission directly from the copyright holder.

To view a copy of this licence, visit http://creativecommons.org/licenses/by/4.0/.

\section{References}

[1] Lum P S, Burgar C G, Van der Loos M, Shor P C, Majmundar M, Yap R. MIME robotic device for upper-limb neurorehabilitation in subacute stroke subjects: A follow-up study. Journal of Rehabilitation Research \& Development, 2006, 43, 631-642.

[2] Hogan N, Krebs H I, Charnnarong J, Srikrishna P, Sharon A. MIT-MANUS: A workstation for manual therapy and training. IEEE International Workshop on Robot and Human Communication, Tokyo, Japan, 1992, 161-165.

[3] Duret C, Courtial O, Grosmaire A G, Hutin E. Use of a robotic device for the rehabilitation of severe upper limb paresis in subacute stroke: Exploration of patient/robot interactions and the motor recovery process. BioMed Research International, 2015, 482389.

[4] Colomer C, Baldoví A, Torromé S, Navarro M D, Moliner B, Ferri J, Noé E.Efficacy of armeo spring during the chronic phase of stroke. Study in mild to moderate cases of hemiparesis. Neurologia, 2013, 28, 261-267.

[5] Takebayashi T, Takahashi K, Amano S, Uchiyama Y, Gosho M, Domen K, Hachisuka K. Assessment of the efficacy of ReoGo-J robotic training against other rehabilitation therapies for upper-limb Hemiplegia after stroke: Protocol for a randomized controlled trial. Frontiers in Neurology, 2018, 9, 730.

[6] Iosa M, Morone G, Fusco A, Bragoni M, Coiro P, Multari M, Paolucci S. Seven capital devices for the future of stroke rehabilitation. Stroke Research and Treatment, 2012, 187965.

[7] Frisoli A, Salsedo F, Bergamasco M, Rossi B, Carboncini M C. A force-feedback exoskeleton for upper-limb rehabilita- tion in virtual reality. Applied Bionics and Biomechanics, 2009, 6, 115-126.

[8] Islam M, Rahmani M, Rahman M. A novel exoskeleton with fractional sliding mode control for upper limb rehabilitation. Robotica, 2020, 38, 2099-2120.

[9] Zeiaee A, Soltani-Zarrin R, Langari R, Tafreshi R. Kinematic design optimization of an eight degree-of-freedom upper-limb exoskeleton. Robotica, 2019, 37, 2073-2086.

[10] Ercolini G, Trigili E, Baldoni A, Crea S, Vitiello N. A novel generation of ergonomic uper-limb wearable robots: Design challenges and solutions. Robotica, 2019, 37, 2056-2072.

[11] Yue Z, Zhang X, Wang J. Hand rehabilitation robotics on poststroke motor recovery. Behavioral Neurology, 2017, 3908135

[12] Tucan P, Vaida C, Plitea N, Pisla A, Carbone G, Pisla D. Risk-based assessment engineering of a parallel robot used in post-stroke upper limb rehabilitation. Sustainability, 2019, 11, 1-28.

[13] Basteris A, Nijenhuis S M, Stienen A H, Buurke J H, Prange G B, Amirabdollahian F. Training modalities in robot-mediated upper limb rehabilitation in stroke: A framework for classification based on a systematic review. Journal of Neuroengineering and Rehabilitation, 2014, 11, 111.

[14] Ceccarelli M, Problems and experiences on cable-based service robots for physiotherapy applications. In: Pisla D, Bleuler H, Rodic A, Vaida C, Pisla A. (eds) New Trends in Medical and Service Robots, Mechanisms and Machine Science, Springer, Cham, Switzerland, 2014, 16, $27-42$.

[15] Hernandez E, Valdez S I, Carbone G, Ceccarelli M. Design optimization of a cable-driven parallel robot in upper arm training-rehabilitation processes. In: Carvalho J, Martins D, Simoni R, Simas H. (eds) Multibody Mechatronic Systems, Mechanisms and Machine Science, Springer, Cham, Switzerland, 2018, 54, 413-423.

[16] Carbone G, Gherman B, Ulinici I, Vaida C, Pisla D. Design issues for an inherently safe robotic rehabilitation device. In: Ferraresi C, Quaglia G, eds., Advances in Service and Industrial Robotics. RAAD 2017. Mechanisms and Machine Science, Springer, Cham, Switzerland, 2018, 49, 1025-1032.

[17] Cafolla D, Russo M, Carbone G. Design and validation of an inherently safe cable-driven assisting device. International Journal of Mechanics and Control, 2018, 19, 23-32.

[18] Cafolla D, Russo M, Carbone G. CUBE, a cable-driven device for limb rehabilitation. Journal of Bionic Engineering, 2019, 16, 492-502.

[19] Laribi M A, Carbone G, Zeghloul S. On the optimal design 
of cable driven parallel robot with a prescribed workspace for upper limb rehabilitation tasks. Journal of Bionic Engineering, 2019, 16, 503-513.

[20] Oyman E, Korkut M, Ylmaz C, Bayraktaroglu Z, Arslan M. Design and control of a cable-driven rehabilitation robot for upper and lower limbs. Robotica, 2021, 1-37.

[21] Laribi M A, Romdhane L, Zeghloul S. Advanced synthesis of the DELTA parallel robot for a specified workspace. In Huapeng Wu (ed.), Parallel Manipulators, Towards New Applications, I-Tech Education and Publishing, Vienna, Austria, 2008, 506

[22] Uicker J J, Pennock G R, Shigley J E. Theory of Machines and Mechanisms, 5th ed., Oxford University Press, New York, USA, 2017.

[23] Flores-Ortiz R, Malta D C, Velasquez-Melendez G. Adult body weight trends in 27 urban populations of Brazil from 2006 to 2016: A population-based study. PLoS ONE, 2019, 14, e0213254.
[24] Major K A, Major Z Z, Carbone G, Pîslă A, Vaida C, Gherman B, Pîslă D L. Ranges of motion as basis for robot-assisted poststroke rehabilitation. Human and Veterinary Medicine, 2016, 8, 192-196.

[25] Martinez C, Tavakoli M. Learning and reproduction of therapist's semi-periodic motions during robotic rehabilitation. Robotica, 2020, 38, 337-349.

[26] Brahmi B, Saad M, Luna C, Archambault P, Rahman M. Passive and active rehabilitation control of human upper-limb exoskeleton robot with dynamic uncertainties. Robotica, 2018, 36, 1757-1779.

[27] Xu Z J, Tian Y T, Li Y. sEMG pattern recognition of muscle force of upper arm for intelligent bionic limb control. Journal of Bionic Engineering, 2015, 12, 316-323.

[28] Ferigo D, Merhi L K, Pousett B, Zhen G X, Menon C. A case study of a force-myography controlled bionic hand mitigating limb position effect. Journal of Bionic Engineering, 2017, 14, 692-705. 